\title{
Influence of Biped Robot's Walking Parameters on its Stability
}

\author{
Zhu Xiaoguang ${ }^{*}$ and Fu Dongxiao
}

School of Energy Power and Mechanical Engineering, North China Electric Power University, Baoding, Hebei, 071000, P.R. China

\begin{abstract}
In the biped robot's walking process, walking parameters have important influence on its stability. In this article we based on the ZMP criterion, using the relationship between ZMP and the support area to judge the walking stability. Calculate the size of stability margin to analyze the influence of parameters' change on stability. By programming in MATLAB and simulating in ADAMS, get the effects of gait parameters (step length, walk cycle, etc) on stability, put forward the reasonable selecting region of parameters, using genetic algorithm to get multiple sets of comprehensive parameters for analysis, the impact of each parameter on stability is identified. It may provide reference for the robot's gait planning later.
\end{abstract}

Keywords: Gait planning, Walking parameter, Walking stability, ZMP.

\section{INTRODUCTION}

Humanoid robot is one of the important research directions in Robotics. Compared with other robots (wheeled, crawler etc.), the humanoid robot has the following characteristics: Discrete supporting foot, alternately touching the ground, walking stability under different circumstances, increased flexibility, more suitable to replace human work in harsh environments; Walking humanoid robot is a high level, strong coupling, nonlinear multi-degree of freedom system and a typical study in control theory and control engineering fields [1]; Walking humanoid robots have anthropomorphic mobile features and better affinities. They are more suitable for the task together with human; Research on walking gait of humanoid robot can promote the development of kinetic prosthetic and rehabilitation medicine; Research on walking humanoid robot could also promote the development of artificial intelligence, bionics, sensors, communications, computers and other related disciplines [2].

The research of biped robot, which includes bionics mechanical engineering, control theory and other disciplines, it is an important branch of robotics research. It has a better mobility, flexibility and adaptability, but its reliability and stability of walking will be worse, so movement stability and reliability have always been one of the main topics and difficult problems in the biped robot research. This paper designs the linear walking gait of the robot, the 3D model of the robot is built in Pro/E, then import into the ADAMS to simulation. Judge the stability of walking by ZMP principle, the influence of the individual changes of the step length, double support time, hip height from the ground, lift height, lateral distance than hip moves and other parameters on the stability of walking is studied, put forward the reasonable selecting region of parameter, using genetic algorithm to get multiple sets of comprehensive and better stability parameters, analyze the data to identify the impact of each parameter on stability, this will help design stable gait.

\section{GAIT PLANNING}

\subsection{Robot Model}

Set each leg of the robot has 6 DOFs(degrees of freedom), the hip has 3,knee has 1, and Ankle has 2. robot's main dimensions: robot's upper body height: $200 \mathrm{~mm}$; the distance between two hip joints: $71 \mathrm{~mm}$; length of upper leg: $90 \mathrm{~mm}$; length of calf: $90 \mathrm{~mm}$; size of sole (length $\times$ width): $120 \times 65$ $\mathrm{mm}$. According to above main dimensions to build 3D model, it will help to the simulation in ADSMS. The parameters are described in Fig. (1):

\subsection{Planning Method}

This article uses parallel gait, when the biped robot is walking, its' two feet are parallel [3]. In the process of walking, the feet always stay parallel to the ground; the motion can be divided into three stages:

(1) Beginning phase: biped robot starts move from upright stationary state, first, the center of gravity fall to suitable height for walking. At the same time by the hip joint's lateral movement, the center of gravity will offset from the middle to the supporting foot.

(2) Normal walking phase: biped robot's leg alternately move forward, the upper body stays upright and projection of center of gravity on the ground moving between two feet, in this process, ZMP should always keep in stable support domain. 

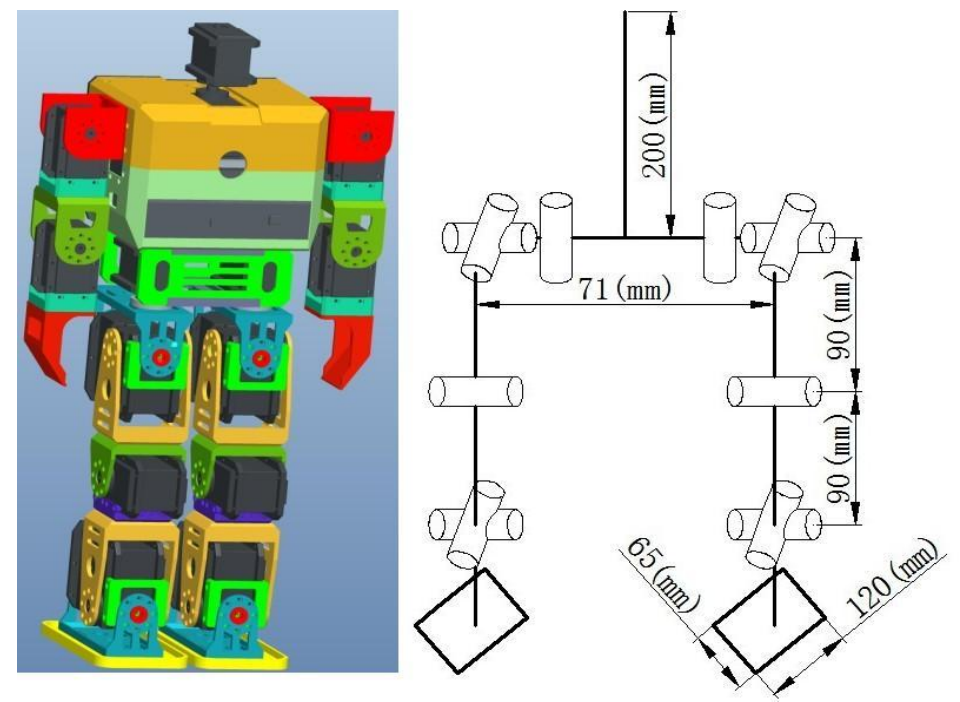

Fig. (1). Robot Dimensions.
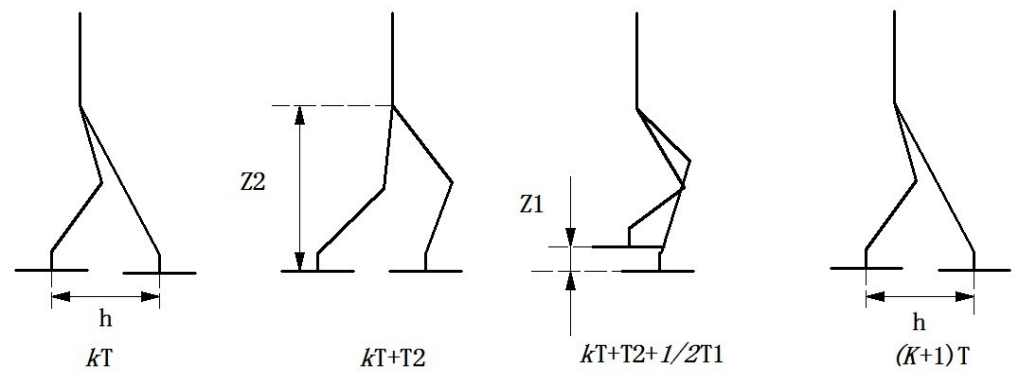

Fig. (2). Robot's walking parameters.

(3) Ending phase: robot's swinging leg stamp half step forward, and then it will be placed in the position of flush with support foot, the center of gravity offset from support foot to middle of two legs. At last raise the robot center of gravity to the initial upright position.

Parameters' initial setting: half a walk cycle T:0.75s; single support time T1:0.5s; double support time T2:0.25s; step $\mathrm{H}: 120 \mathrm{~mm}$; lift height $\mathrm{Z1}: 20 \mathrm{~mm}$, hip height from the ground Z2:160 mm, lateral distance of hip motion Y:30 mm.

The parameters are described in Fig. (2):

When the robot's walking motion is planned, its walking trajectory will be planned, in this paper, we ignore the coupling between forward motion and lateral motion and carry out resolution on movements, in their own plane to plan the trajectory of hip and ankle, Use polynomial interpolation method to solve the trajectory, then with the knowledge of robot inverse kinematics, the joint angle during walking can be obtained [4].

\section{THE STABILITY CRITERION}

In this paper, we use the ZMP (zero moment point) principle proposed by the Yugoslav scholars, Vukobratovic and Stepanenko to judge the walking stability [5-6]. It represents force projection point on the ground of biped robot suffered force of gravity and inertia force during movement, at this point the force moment is zero. When walking on the hori- zontal ground, ZMP point must always in the projection of convex area consist by support feet on the ground, which is stable support domain. In the process of walking, the minimum distance between ZMP point and stable support area boundary is defined as stability margin, greater stability margin is, that is closer ZMP is to the center of the support polygon, biped robot will more stable. ZMP formula:

$$
\begin{aligned}
& Y_{z m p}= \frac{\sum_{i=1}^{n} f_{i} Y_{i}}{\sum_{i=1}^{n} f_{i}}=\frac{\sum_{i=1}^{n} m_{i}\left(\ddot{x}_{i}+g\right) y_{i}-\sum_{i=1}^{n} m_{i} \ddot{y}_{i} x_{i}}{\sum_{i=1}^{n} m_{i}\left(\ddot{x}_{i}+g\right)} \\
& Z_{z m p}=\frac{\sum_{i=1}^{n} f_{i} Z_{i}}{\sum_{i=1}^{n} f_{i}}=\frac{\sum_{i=1}^{n} m_{i}\left(\ddot{x}_{i}+g\right) z_{i}-\sum_{i=1}^{n} m_{i} \ddot{z}_{i} x_{i}}{\sum_{i=1}^{n} m_{i}\left(\ddot{x}_{i}+g\right)}
\end{aligned}
$$

According to above method for programming in MATLAB and draw the ZMP curve of a walk cycle, this is shown in Fig. (3):

The right foot is the staring origin, move left foot supported by right foot forward, and then the center of gravity is moved to left foot, next left foot support right foot to take a step. In (Fig. 3), The biggest convex area is stable support domain, which is composed of the point between supporting foot and ground, the solid line is the center line of stable 


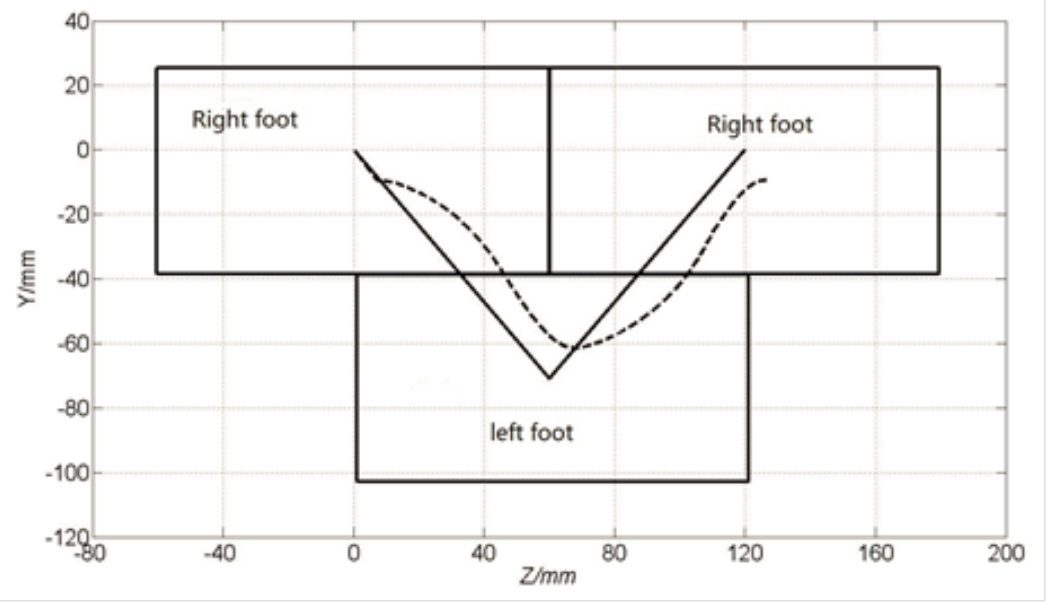

Fig. (3). Actual ZMP curve and center line of support domain.

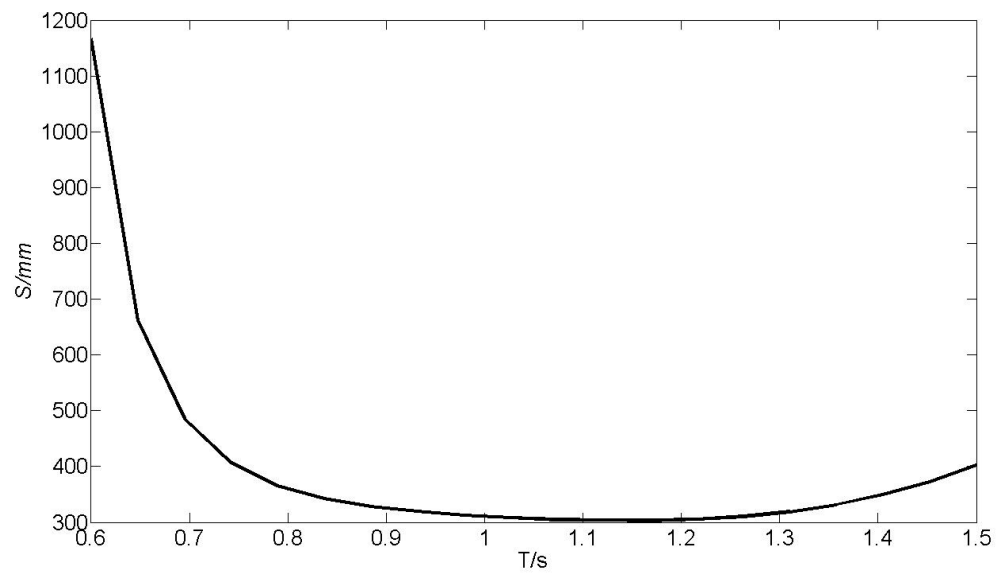

Fig. (4). T-S curve.

support domain, the dotted line is actual ZMP curve calculated by above parameters, the distance between two lines is defined $\mathrm{S}$, it is the reference to judge the walking stability. Smaller the $\mathrm{S}$ value is, higher the contact ratio of actual ZMP curve and center line of support region is, greater stability margin is, more stable when robot is walking, $\mathrm{S}$ is calculated as shown in Equation 2-3.

$\mathrm{S}=\sum_{\mathrm{K}=1}^{\mathrm{N}} \sqrt{\left(Z_{z m p}(\mathrm{k})-Z_{d z m p}(\mathrm{k})\right)^{2}+\left(\mathcal{Y}_{z m p}(\mathrm{k})-y_{d z m p}(\mathrm{k})\right)^{2}}$

Among them, $\mathrm{K}$ is the sampling points, $\left(Z_{d z m p}, y_{d z m p}\right)$ is the point on the center line of support domain, ( $\left.Z_{z m p}, y_{d z m p}\right)$ is the point on actual ZMP line [7-8].

\section{THE EFFECTS OF GAIT PARAMETERS ON STA- BILITY}

Select the parameters T1, T2, H, Z1, Z2, Y as the research subjects, in order to analyze the effects of gait parameters on stability. First, consider the influence on stability while change a parameter, we don't consider temporarily the coupling relationship between each parameter, use $\mathrm{S}$ as the reference to judge the walking stability. Each parameter as the abscissa value, $\mathrm{S}$ as the ordinate value, two dimension curve graphs will be drew.

\subsection{Influence of Half a Walk Cycle T}

The effects of half a walk cycle on stability is mainly reflected in the ratio of single support and double support time, in this paper the single time remain the same, just change $\mathrm{T}$ value, that will change their ratio. As can be seen from (Fig. 4), with $\mathrm{T}$ value increases, $\mathrm{S}$ value decreases rapidly to a steady value and then increases. Take into account the proportion of double support time is too large, the robot's walking efficiency will be reduced, the range of optimal T2 and T1 ratio.

$$
\frac{\mathrm{T} 2}{T 1}=[0.5 \sim 1]
$$

\subsection{Influence of Step Length $H$}

As can be seen from (Fig. 5), with the increase of $\mathrm{H}, \mathrm{S}$ value decreases firstly then increases, walking is tending 


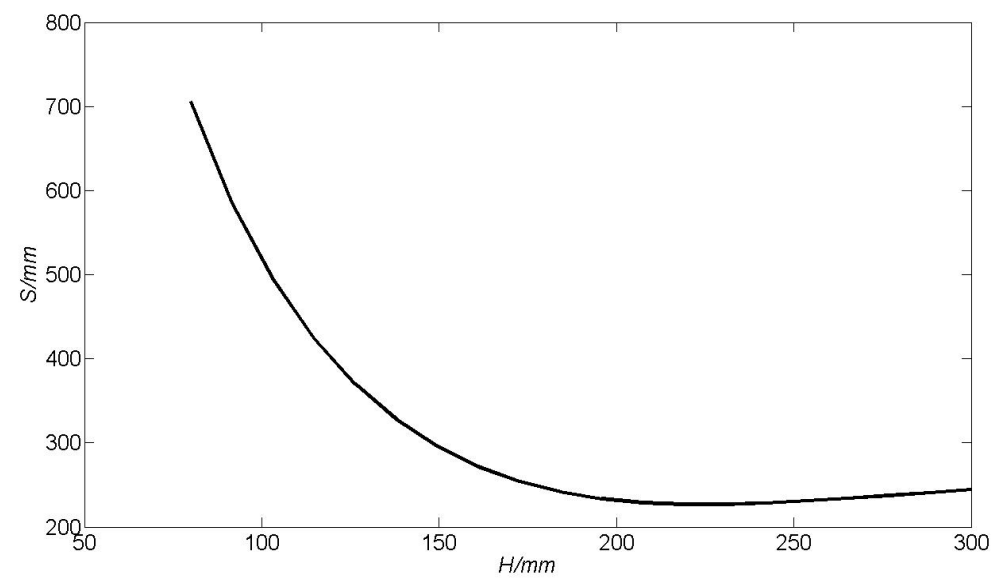

Fig. (5). H-S curve.

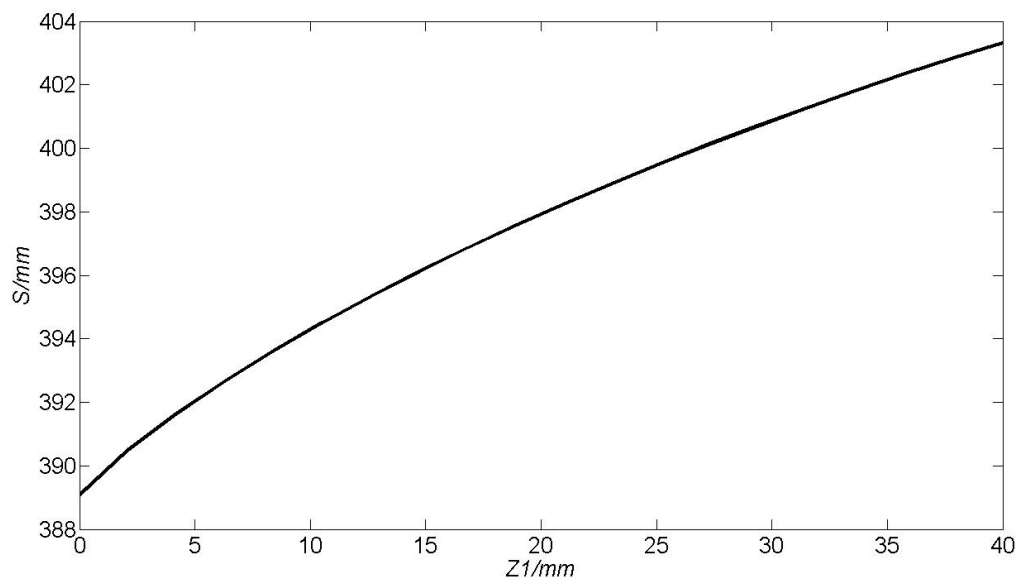

Fig. (6). Z1-S curve.

towards stability, but the step length $\mathrm{H}$ is affected by robot structure, it can't increases infinitely. Under the condition of robot structure allows, increase the step length of robot appropriately can improve the walking stability.

\subsection{Influence of Lift Height Z1}

As can be seen from (Fig. 6), while lift height is changed, the $\mathrm{S}$ value changes in a small scope. This shows lift height has little impact on the stability. But with the increase of lift height, the torque of each joint will increases, Energy consumption will also increase, so we should choose a smaller lift height under the condition of walking stability.

\subsection{Influence of Hip Height $\mathrm{Z2}$}

As can be seen from (Fig. 7), with the analysis of lift height is similar, hip height has little impact on the stability. But consider from other aspects, reduce the hip height, the center of gravity will also be reduced, this will be good to maintain stability.

\subsection{Influence of the Lateral Distance that Hip Moves $Y$}

As can be seen from the (Fig. 8), with the increase of lateral distance that hip moves, $\mathrm{S}$ value first decreases and then increases. So when plan the robot's gait, choose the right distance is helpful to increase the walking stability.

\section{OPTIMAL WALKING ROBOT PARAMETERS WITH GENETIC ALGORITHMS}

Above we only consider the influence of individual parameters on walking stability; next we will find multiple sets of comprehensive gait parameters under different walking speed by genetic algorithm to analysis.

Genetic algorithm is a high efficient and parallel globally random search optimization method which simulates the evolutionary mechanism of nature, it simulates natural selection, reproduction, crossover and mutation phenomenon occurred in natural genetic process. It reserves a set of 


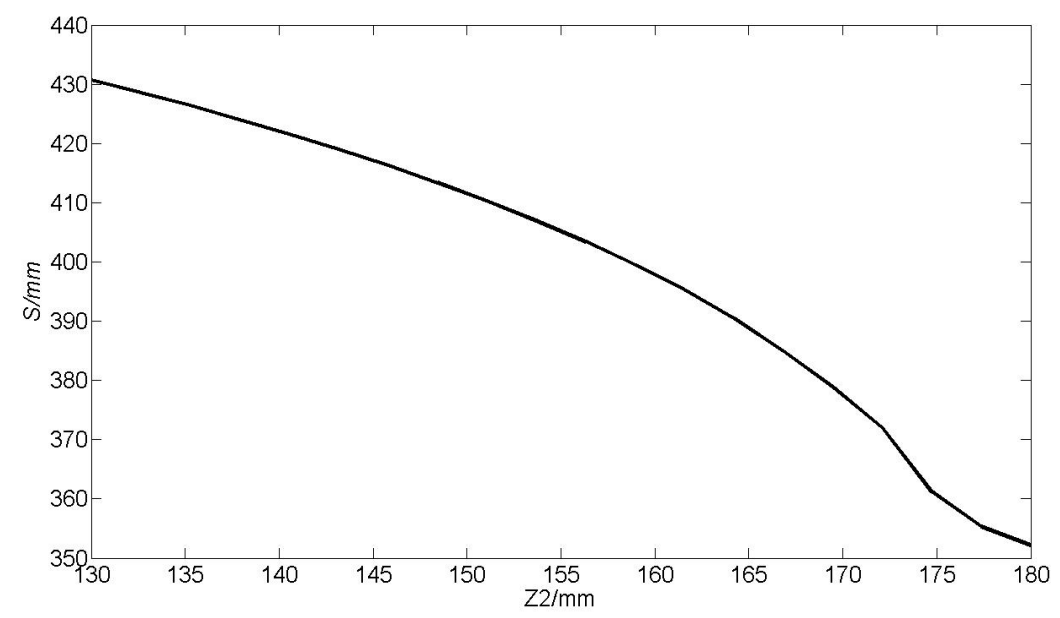

Fig. (7). Z2-S curve.

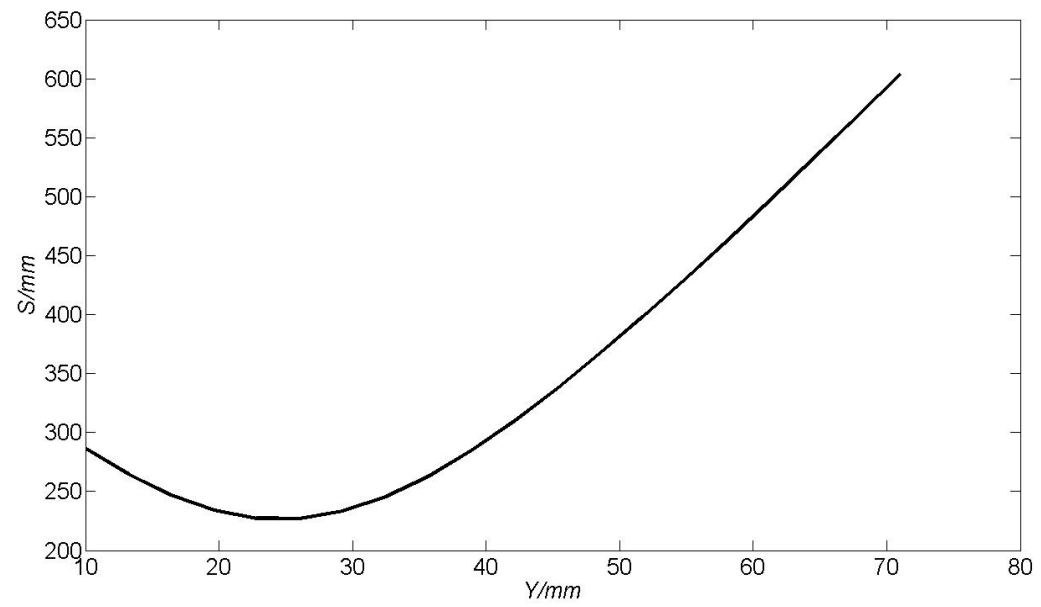

Fig. (8). Y-S curve.

Table 1. Tuning parameters under different speed.

\begin{tabular}{|c|c|c|c|c|c|c|}
\hline $\mathrm{V} /(\mathrm{mm} \cdot \mathrm{s}-1)$ & $\mathbf{T} / \mathbf{s}$ & $\mathbf{H} / \mathbf{m m}$ & $\mathrm{Y} / \mathrm{mm}$ & $\mathrm{Z1} / \mathrm{mm}$ & $\mathrm{Z2} / \mathrm{mm}$ & $\mathrm{S} / \mathbf{m m}$ \\
\hline $\mathrm{V} 1=80.04$ & 0.99956 & 80 & 30.3294 & 20 & 160 & 166.1283 \\
\hline$V 3=133.90$ & 0.89619 & 120 & 32.7586 & 20 & 160 & 89.4552 \\
\hline $\mathrm{V} 4=157.61$ & 0.88827 & 140 & 33.1056 & 20 & 160 & 71.8102 \\
\hline$V 6=203.55$ & 0.88431 & 180 & 33.3138 & 20 & 160 & 53.2464 \\
\hline$V 7=238.94$ & 0.83284 & 200 & 33.7996 & 20 & 160 & 46.6049 \\
\hline
\end{tabular}

candidate solution after each iteration, according to some indicators (objective function) to select the better individuals from the solution group, using genetic operators (selection, crossover and mutation) to combine these individuals, produce a new generation of candidate solutions group, repeat this process until the objective function is satisfied. By program in MATLAB, different walking speed is taken as vari- able to get multiple sets of optimization parameters as shown in Table 1:

The range of genetic algorithm optimization parameter as shown in Table 2:

Use each parameter to plot the ZMP curve as shown in (Fig. 9): 
Table 2. Genetic algorithm optimization parameter range.

\begin{tabular}{|c|c|c|c|c|c|}
\hline & $\mathbf{T} / \mathbf{s}$ & $\mathbf{H} / \mathbf{m m}$ & $\mathbf{Y} / \mathbf{m m}$ & $\mathbf{Z 1} / \mathbf{m m}$ & $\mathbf{Z 2} / \mathbf{m m}$ \\
\hline \hline the minimum value & 0.55 & 80 & 0 & 20 & 140 \\
\hline the maximum value & 1.5 & 200 & 71 & 40 & 160 \\
\hline
\end{tabular}

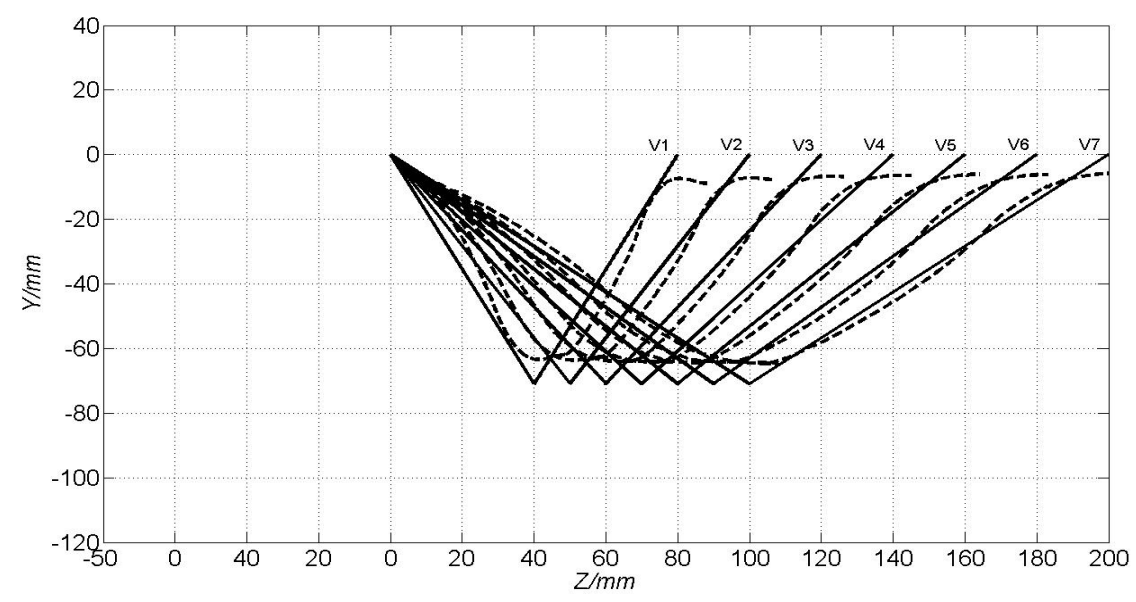

Fig. (9). ZMP curve under the optimal parameters.

As can be seen from (Fig. 9), the optimum ZMP curve under different speed and center line of the support domain have high coincidence degree, $\mathrm{S}$ value under different speed compares to the original S $(235.4643 \mathrm{~mm})$ has a large decrease, according to the concept of stability margin, robot's stability is improved greatly. From the variation and range of optimal parameters can be seen:

(1) T value between $0.8-1 \mathrm{~s}$, this shows that the range of $\mathrm{T} 1 / \mathrm{T} 2$ value is credible.

(2) Compare H value under different speed, with its' increase, $\mathrm{S}$ value is reduced, it shows that increase $\mathrm{H}$ value rationally in the suitable scope can improve stability of walking.

(3) $\mathrm{S}$ value is in a middle position, this is consistent with the influence of a single parameter.

(4) Z1, Z2 value is a smaller value under the range, it similar to the third conclusion.

Select the parameters in speed V3 to calculate in MATLAB, then we can get a set of joint angle, import robot's 3D model into ASAMS to simulation, the result is shown in (Fig. 10).

The simulation experiment shows robot has a stability walking gait, the effect is good and the process of calculate is feasible.

For analyze the influence of various parameters on the walking stability in detail, the regression analysis of the data is calculated by GA can get the results as shown in Table 3 .
As can be seen from table $\mathbf{3}$, the regression model is: 382.467-235.122*T-0.585*H+3.289*Y+0.11*Z2-0.468*Z1, this equation shows that T,H,Z2 is proportional to $S$. Y,Z1 are inversely proportional to $S$. The coefficients of $Z 1$ and Z2 satisfy the above laws. GA is a constant optimal process; the data will always evolve to a particular direction. T, H, Y will not change in the direction of maximum. So the coefficients of T, H, Y also meet above rules, their value is large, the coefficients of $\mathrm{Z1}$ and $\mathrm{Z} 2$ are small, the coefficient of $\mathrm{T}$ is significantly larger, for its value varies between 0.55 and 1 , compared with other parameter, its absolute value is very small, $\mathrm{T}$ value has a small undulation can cause a big change to $\mathrm{S}$ value, so the coefficient of $\mathrm{T}$ is larger than others. Compare the partial correlation coefficients of each parameter, $\mathrm{T}$, $\mathrm{H}$ and $\mathrm{Y}$ is larger, we may draw a conclusion that the values of $\mathrm{T}, \mathrm{H}$ and $\mathrm{Y}$ have great influence on walking stability, while $\mathrm{Z} 1$ and $\mathrm{Z} 2$ are smaller.

\section{CONCLUSION}

This article based on the ZMP criterion to design a method that obtain maximal stability margin to judge walking stability, research the influence of individual parameters on walking stability, get the best comprehensive gait parameters under different walking speed by genetic algorithm, then prove the law of influence is credible by the data analysis, analyze the influence of various parameters on the walking stability. It may provide reference for the robot's steady gait planning. 


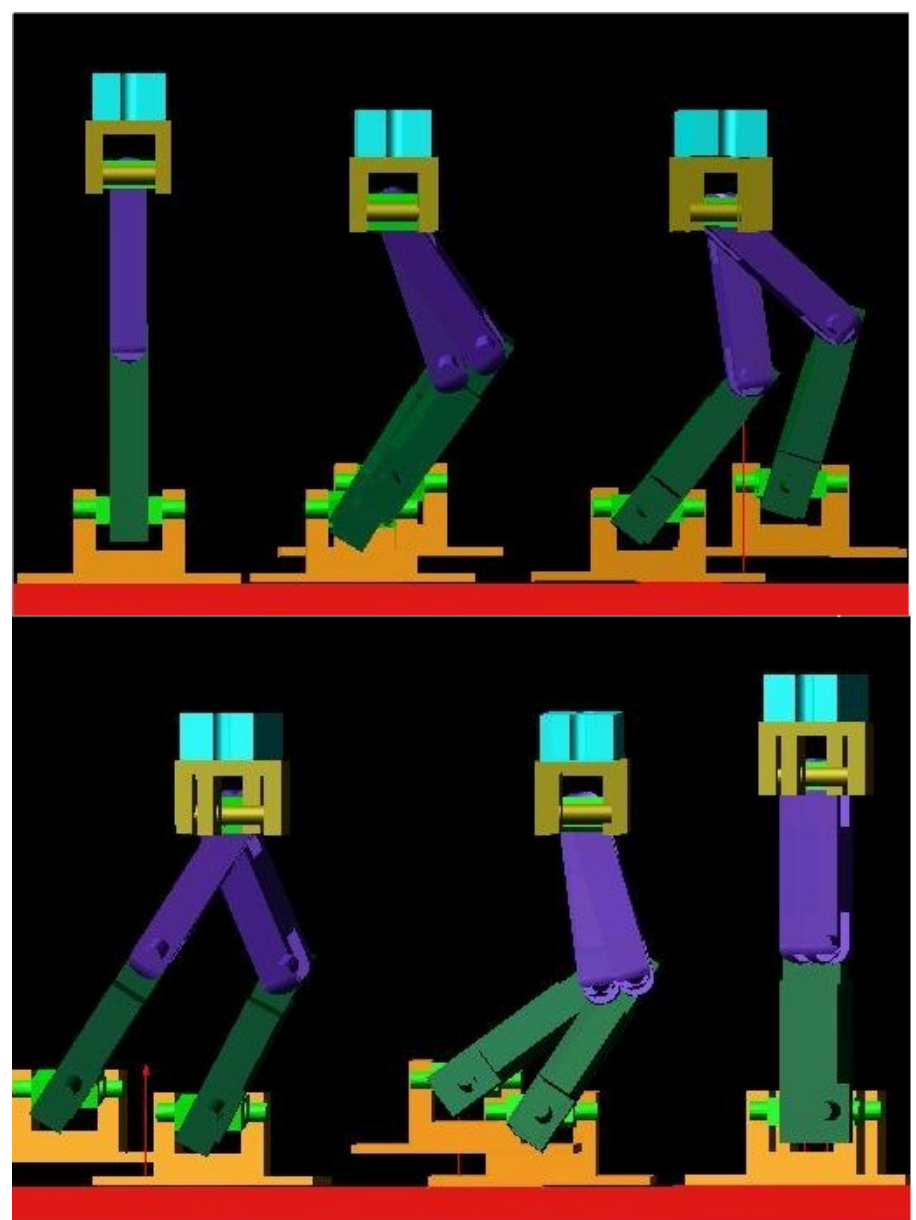

Fig. (10). ADAMS simulation diagram.

Table 3. data regression analysis.

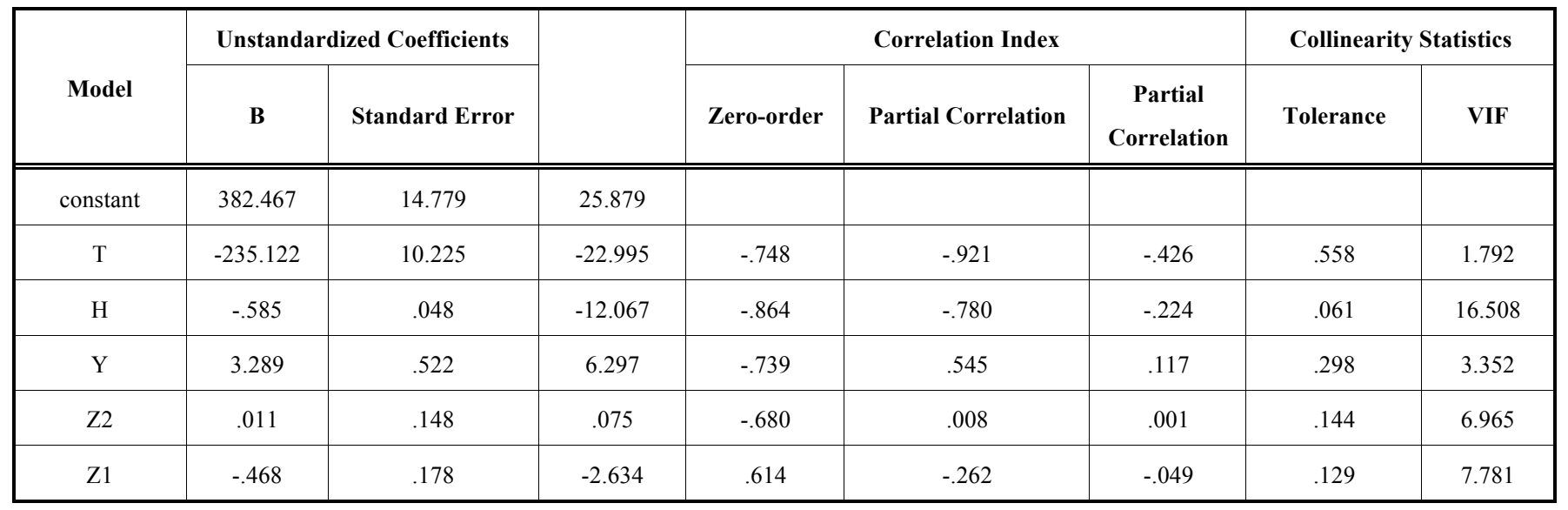

\section{CONFLICT OF INTEREST}

The author confirms that this article content has no conflict of interest.

\section{ACKNOWLEDGEMENTS}

This article is supported by "the Fundamental Research Funds for the Central University”, No.13QN57.

\section{REFERENCES}

[1] S. Aoi, and K. Tsuchiya, "Generation of bipedal walking through interactions among the robot dynamics, the oscillator dynamics, and the environment: Stability characteristics of a five-link planar biped robot," Autonomous Robots, vol. 30, pp. 123-141, February 2011.

[2] R. Yared, X. Défago, J. Iguchi-Cartigny, and M. Wiesmann, "Collision prevention platform for a dynamic group of asynchronous cooperative mobile robots," Journal of Networks, vol. 2, pp. 28-39, February 2007. 
[3] J. Ji, "HIT-II biped walking robot gait planning study," $\mathrm{PhD}$. thesis, Harbin industrial university Harbin, China, 2000.

[4] Z. Cai, Robotics, Tsinghua university press: Beijing, 2000.

[5] D. Yang, J. Wang, and L. Niu, "Gait planning of humanoid robot based on ZMP," Robot, vol. 23, pp. 504-508 June 2001.

[6] G. Xuan, and R. Cheng, Genetic algorithms and engineering optimization. Tsinghua university press: Beijing, 2004.
[7] C. Fang, "The Dynamic analysis of the robot based on ADAMS virtual console," M.S. thesis, Beijing University of Posts and Telecommunications. Beijing, China, 2009.

[8] W. Zhang, and C. Kuang, SPSS statistical analysis based tutorial. Higher education press: Beijing, 2001.

Received: September 22, 2014

(C) Xiaoguang and Dongxiao; Licensee Bentham Open.

This is an open access article licensed under the terms of the Creative Commons Attribution Non-Commercial License (http://creativecommons.org/licenses/by-nc/3.0/) which permits unrestricted, non-commercial use, distribution and reproduction in any medium, provided the work is properly cited. 